Provided for non-commercial research and education use. Not for reproduction, distribution or commercial use.

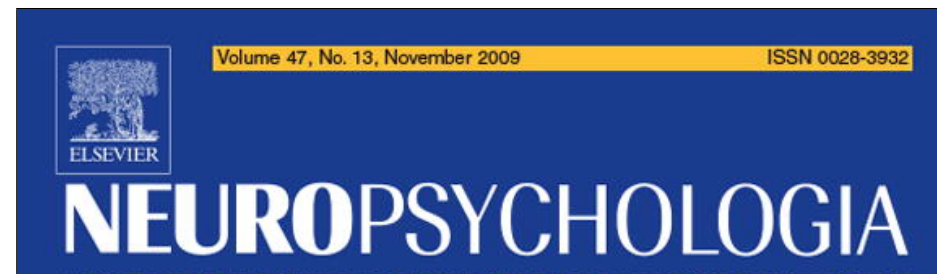

An international journal in behavioural and cognitive neuroscience

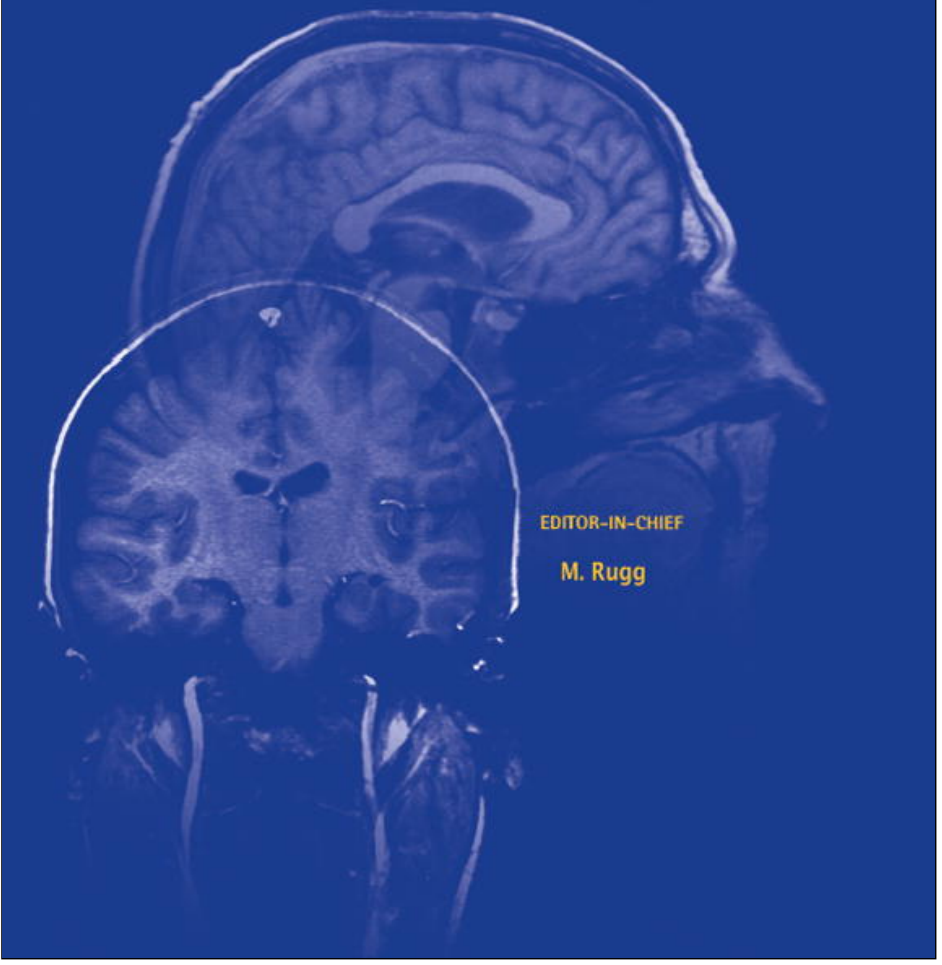

This article appeared in a journal published by Elsevier. The attached copy is furnished to the author for internal non-commercial research and education use, including for instruction at the authors institution and sharing with colleagues.

Other uses, including reproduction and distribution, or selling or licensing copies, or posting to personal, institutional or third party websites are prohibited.

In most cases authors are permitted to post their version of the article (e.g. in Word or Tex form) to their personal website or institutional repository. Authors requiring further information regarding Elsevier's archiving and manuscript policies are encouraged to visit:

http://www.elsevier.com/copyright 


\title{
Exploring multimodal semantic control impairments in semantic aphasia: Evidence from naturalistic object use
}

\author{
Faye Corbett ${ }^{\mathrm{a}}$, Elizabeth Jefferies ${ }^{\mathrm{b}}$, Matthew A. Lambon Ralph ${ }^{\mathrm{a}, *}$ \\ a University of Manchester, UK \\ ${ }^{\mathrm{b}}$ University of York, UK
}

\section{A R T I C L E I N F O}

\section{Article history:}

Received 7 January 2009

Received in revised form 14 May 2009

Accepted 27 May 2009

Available online 3 June 2009

\section{Keywords:}

Stroke aphasia

Semantic memory

Semantic cognition

Non-verbal

Object use

Naturalistic action

\begin{abstract}
A B S T R A C T
Semantic processing can break down in qualitatively distinct ways in different neuropsychological populations. Previous studies have shown that patients with multimodal semantic impairments following stroke - referred to as semantic aphasia (SA) - show deficits on a range of conceptual tasks due to a failure of semantic control processes in the context of prefrontal and/or temporoparietal infarction. Although a deficit of semantic control would be expected to impair performance in all modalities in parallel, most previous research in this patient group has focussed primarily on tasks employing words. This study explored the consequences of deregulated semantic cognition for an indisputably non-verbal task-naturalistic object use. Patients with SA performed more poorly than control participants on a range of everyday tasks assessed by the Naturalistic Action Test (NAT, Schwartz, M. F., Buxbaum, L. J., Ferraro, M., Veramonti, T., \& Segal, M. (2002). Naturalistic action test. Thames Valley Test Company). Moreover, their scores on this assessment correlated with those obtained on language-based semantic tasks, suggesting that a common deficit could underlie the impairment in both modalities. As previously observed in the verbal domain, performance on the NAT was poorer when control processes were taxed by dual-task situations and the inclusion of semantically related distracting objects. A number of characteristics of the patients' action sequences were specifically indicative of deregulated semantic cognition. Their everyday action sequences were highly fragmented by a tendency to abandon subtasks before their completion and engage, instead, in extended periods of aimless "toying" with objects. The patient group also exhibited recurrent perseverative behaviour. These findings parallel the performance of a recurrent connectionist model of naturalistic action developed by Botvinick and Plaut [Botvinick, M. \& Plaut, D. C. (2004). Doing without schema hierarchies: A recurrent connectionist approach to normal and impaired routine sequential action. Psychological Review, 111, 395-429], after the mechanism responsible for controlling action in a temporally sensitive manner was damaged. This study provides converging evidence for a failure of control processes underlying semantic memory impairment in SA, which is reflected not only in patients' performance on language-based tasks, but also in the non-verbal domain of naturalistic object use.
\end{abstract}

(C) 2009 Elsevier Ltd. All rights reserved.

\section{Introduction}

Semantic cognition encompasses the processes and representations underlying our knowledge and use of the meanings of words, pictures, objects, sounds and faces (Jefferies \& Lambon Ralph, 2006; Lambon Ralph, Lowe, \& Rogers, 2007; Rogers et al., 2004). When semantic cognition becomes damaged there are serious consequences for many activities of daily living-ranging from holding conversations with others to coordinating everyday tasks such as making a cup of coffee. Neuropsychological studies have

\footnotetext{
* Corresponding author at: Neuroscience and Aphasia Research Unit (NARU), School of Psychological Sciences, Zochonis Building, University of Manchester, Oxford Road, Manchester, M13 9PL, UK.
}

E-mail address: matt.lambon-ralph@manchester.ac.uk (M.A. Lambon Ralph). established three distinct ways in which semantic cognition can become impaired in different patient groups: (1) representations can become degraded or distorted as in semantic dementia and herpes simplex virus encephalitis (Hodges, Patterson, Oxbury, \& Funnell, 1992; Kapur et al., 1994; Lambon Ralph et al., 2007; Snowden, Goulding, \& Neary, 1989); (2) access to intact representations can become impaired through damage to connections in and out of the semantic store, as seen in the modality specific deficits such as visual agnosias and word deafness (Farah, 2004; Franklin, Turner, Lambon Ralph, Morris, \& Bailey, 1996); or, (3) failure of top-down control over activation within the semantic store can cause processing of the meaning to become deregulated. Because we know so many things relating to different aspects of a single concept, it is critical to shape activation in order to produce task, time and context-appropriate behaviours. Taking the concept 'piano' as an example, the facets of knowledge pertinent for its canonical 
use as a musical instrument differ substantially from those that must be retrieved when moving the object around (Saffran, 2000). Moreover, the critical aspects of meaning can change over time for the same concept. Consider the multiple roles that a knife plays while making a cheese and chutney sandwich: opening packets, spreading butter, cutting bread, slicing cheese, scooping chutney, etc., all of which require different aspects of the knife's properties to be brought to the fore, including different ways of holding and manipulating the object, while its canonical function (i.e., cutting) must be inhibited for most of the task (Noonan et al., in press). Disorders of semantic control have been observed in a subset of patients with stroke aphasia; the nature of this impairment, however has not been the focus of much previous neuropsychological research.

Semantic dementia (SD) is a progressive neurological disorder characterised by a highly selective deficit of semantic memory that is associated with relatively circumscribed bilateral atrophy of the anterior temporal lobes (ATL) especially in inferior and lateral aspects (Mummery et al., 2000; Nestor, Fryer, \& Hodges, 2006). In this condition, conceptual knowledge is affected in both verbal and non-verbal domains across a broad range of modalities, including spoken and written words, pictures, smells, environmental sounds and touch (Bozeat, Lambon Ralph, Patterson, Garrard, \& Hodges, 2000; Coccia, Bartolini, Luzzi, Provinciali, \& Lambon Ralph, 2004; Luzzi et al., 2007). Further, patients with SD show strong correlations between tasks and consistently fail the same items irrespective of changes in task parameters and modality (Bozeat et al., 2000; Jefferies \& Lambon Ralph, 2006). Other aspects of cognition, in contrast, are well-preserved in this group; patients score within the normal range on tests of visuo-spatial and attentional/executive processing, they have good memory for recent events and speech is fluent, syntactically correct and largely free from phonological errors (Hodges et al., 1992; Snowden et al., 1989). The highly stable nature of multimodal semantic impairment exhibited by patients with SD suggests that the ATL - atrophied in this group - acts as a repository of amodal semantic knowledge, which progressively and permanently degrades in this condition (Jefferies \& Lambon Ralph, 2006; Rogers et al., 2004). This selective and multimodal semantic deficit has also been replicated using rTMS over the lateral ATL in normal participants (Lambon Ralph, Pobric, \& Jefferies, 2009; Pobric, Jefferies, \& Lambon Ralph, 2007).

Multimodal semantic impairments are also observed in a subset of patients with aphasia following stroke (Chertkow, Bub, Deaudon, \& Whitehead, 1997; Jefferies \& Lambon Ralph, 2006); a condition hereafter referred to as semantic aphasia (SA). As observed in SD, patients with SA perform poorly on a range of semantic tasks that tap different input/output modalities. When matching semantically associated concepts, for example, patients obtain scores outside the normal range when stimuli are presented either as words or pictures (Jefferies \& Lambon Ralph, 2006). A sub-type of aphasia known as transcortical sensory aphasia (TSA) most closely resembles the neuropsychological profile seen in SD. Multimodal semantic impairment in TSA leaves other aspects of language including repetition and speech fluency - intact. Despite these similar neuropsychological profiles, the patterns of underlying brain damage in SD and SA are quite different: while SD is the result of atrophy largely restricted to the ATL, SA occurs in the context of damage to the left inferior prefrontal (LIPC) and/or temporoparietal cortex (Berthier, 2001; Chertkow et al., 1997; Hart \& Gordon, 1990; Jefferies \& Lambon Ralph, 2006; Saygin, Dick, Wilson, Dronkers, \& Bates, 2003).

It is perhaps due to the very different patterns of neurological damage in SA and SD that these semantic impairments have largely been explored by separate neuropsychological literatures. Jefferies and Lambon Ralph (2006) were the first to directly compare SD and SA to determine if the distinct profiles of neurological damage would manifest in qualitatively different semantic deficits (see also Jefferies, Baker, Doran, \& Lambon Ralph, 2007; Jefferies, Patterson, \& Lambon Ralph, 2008). Although SA and SD patients failed the same range of picture and word-based semantic tasks, there were clear differences in the ways in which semantic cognition was impaired. Consistent with previous findings, ATL atrophy in SD was associated with a pattern of impairment suggestive of degraded knowledge. While cognition was generally intact in this group, patients displayed a central semantic impairment that was impervious to changes in task demands and modality. A contrasting profile of impairment observed in SA indicated that these patients had not lost their conceptual knowledge but a failure of control processes had caused semantic cognition to become deregulated. As a consequence, SA patients' performance on a battery of semantic tasks was: (a) much less consistent across tasks than observed in SD; (b) characterised by associative semantic errors in picture naming (e.g., squirrel-"nuts"), which were not made by the SD patients; (c) improved or degraded following constraining or distracting cues in picture naming; and (d) sensitive to the control requirements of each trial. Because a degree of control is required in all tasks, SA patients' deficit of conceptual knowledge was persistent across tests tapping different input/output modalities.

Impaired semantic cognition in the context of SD and SA implicates a distributed neural network, covering frontal, temporal and parietal regions. Moreover, the degraded nature of conceptual representations in SD suggests that the ATL bilaterally has a specific role as a repository of amodal semantic information, while the left inferior prefrontal cortex and temporoparietal areas - damaged in SA - work to control activation within this store. These findings converge with functional neuroimaging studies which report activation in the same network of regions when healthy participants engage in semantically demanding tasks, at least when both PET and fMRI are taken into account (Devlin et al., 2000; Visser et al., submitted-a, submitted-b). Of the cortical areas damaged in $S A$, the left inferior frontal gyrus is commonly found to react to the demand placed on control processes by semantic tasks (e.g., Thompson-Schill, D’Esposito, Aguirre, \& Farah, 1997). A growing body of evidence suggests that left inferior parietal and posterior middle temporal areas can also show sensitivity to this factor. This region is more highly activated when different associations must be retrieved from the same concept, or when target responses are weakly associated with a probe or featured as part of a large array of distracters (e.g., Demb et al., 1995; Gold \& Buckner, 2002; Noppeney, Phillips, \& Price, 2004; Thompson-Schill et al., 1997; Wagner, PareBlagoev, Clark, \& Poldrack, 2001). Based on this evidence, it seems likely that the left inferior prefrontal cortex and posterior temporoparietal area work in tandem to control semantic processing; a proposal that is strengthened by the presence of substantial whitematter connections between these two regions via the arcuate and superior longitudinal fasciculi (Gloor, 1997; Parker et al., 2005). Furthermore, patients with damage to different areas of this network have been found to have virtually indistinguishable neuropsychological profiles by Berthier (2001) and Jefferies and Lambon Ralph (2006).

Uncovering multimodal semantic impairment in the context of SA is a striking finding given that patients are typically studied in the domain of their most prominent presenting symptom-language impairment. By extending into the object use domain, a recent study provided converging evidence for non-verbal semantic impairment in SA (Corbett et al., in press), which was previously only documented using word-based tasks (Jefferies \& Lambon Ralph, 2006). Patients with SA and SD were assessed on a battery of tasks tapping knowledge relating to 36 household items-including demonstrating the normal use of those objects. Both groups displayed deficits of non-verbal semantic knowledge but the nature of those impairments differed qualitatively in line with degraded 
or deregulated semantic cognition in SD and SA respectively. As observed in the verbal domain, the SD patients exhibited highly consistent impairment for the same items across tasks, irrespective of whether they were demonstrating an object's use or employing another aspect of knowledge relating to that item (see Bozeat, Lambon Ralph, Patterson, \& Hodges, 2002 for original data). The SA patients did not display the same degree of consistency; their performance was dependent on the demand placed on control processes by the different tasks-even when those tasks tapped knowledge relating to the same items. Straightforward object use demonstration, for example, was relatively intact in SA patients, in comparison to much poorer performance on an executively demanding, mechanical-puzzles task. Similarly, while SA patients found it relatively easy to match the same item presented in different modalities (e.g., word-picture matching), their ability to match different items with a shared attribute was much poorer. In demonstrating normal object-use, the SD group were uniquely sensitive to item frequency, which reflected their degrading core representations. The SA patients did not show the same effect. Further, the groups' different sources of damage to semantic cognition were evident in the types of errors they made during object use; while SD patients made mostly omission errors, the SA group also made a number of erroneous intrusion errors that were unrelated to the target action.

At present, the behavioural consequences of impaired semantic control are incompletely understood with only a few previous studies addressing this issue directly in SA. Although Corbett et al. (in press) assessed the non-verbal aspect of semantic processing, the single object use tasks employed in that study only placed minimal demands on control processes. The aim of the present study was to gain further insight into the effect of deregulated semantic cognition in the non-verbal domain. The Naturalistic Action Test (NAT, Schwartz, Buxbaum, Ferraro, Veramonti, \& Segal, 2002) was selected to assess SA patients' non-verbal abilities, for two major reasons. First, from a neuropsychological perspective, this test places a high demand on the controlled use of semantic knowledge; participants must not only draw on their understanding of the purpose of everyday objects but also coordinate that knowledge in a sequence of actions. Two features of the NAT specifically manipulate the need to shape semantic activation carefully. Participants are required to: (1) manage situations in which a task has a dual-goal, such as making a cup of coffee as well as a slice of toast, and (2) exclude semantically related distracter objects embedded in the array of test materials. By placing sizeable demands on the aspects of cognition damaged in SA, this task gives unique insight into the implications of deregulated semantic cognition beyond the limitations of previous studies.

The second reason for selecting the NAT was theoretical. Extended, sequential object use is an exceedingly rich and complex domain and, as a result, is a difficult topic to describe, investigate and generate theories about. The NAT, however, provides a formal method of assessment and, more importantly, has been the focus of two major computational models (e.g., Botvinick \& Plaut, 2004; Cooper, Schwartz, Yule, \& Shallice, 2005; Cooper \& Shallice, 2000, see Section 5). These provide an invaluable framework not only to score but also to consider the broader implications and potential computational implementations of cognitive control. For the purposes of the present study we will concentrate on a recurrent connectionist model developed by Botvinick and Plaut (2004). The simple network structure of this model, which generates action sequences over time, makes minimal assumptions about the underlying processing system, rendering it particularly suitable for modelling patient data. Moreover, the model makes predictions about the impact of different degrees of damage, which can be compared with patients of varying impairment severity.
The model's architecture consists of three layers. The first represents a perceptual input, either visual or praxic, received when the system focuses attention on a particular object. The second layer of 'hidden' units transforms the perceptual input into an appropriate action, which is generated by the third layer. The response output modifies the environment and the perception-action loop cycles again. Critically for naturalistic action, the system's hidden layer features recurrent connections that maintain information over time, enabling the system to act in a temporally sensitive manner-selecting appropriate outputs based on actions that have gone before. Following training, the model successfully reproduced four coffee-making sequences. Action was managed flexibly by the model, which was able to vary the order of different subtasks (e.g., add milk/add sugar) as well as substitute equivalent subtasks for one another (e.g., add sugar from packet/add sugar from bowl) and cope with hidden environmental states (e.g., sugar already added/not yet added to the cup). The model was also able to distinguish between similar everyday action sequences (i.e., make coffee/make tea).

Having successfully reproduced everyday action sequences, the model was impaired at two levels of severity. Firstly, a mild degree of damage was implemented to replicate action slips, which commonly interrupt healthy participants' everyday action. Action slips are thought to occur due to cross-talk from task irrelevant cognitive activity, which disrupts an internal representation of temporal context. The functional correlate of mental distraction was implemented in the model with random noise added to activation values in the system's recurrent connections. Under low levels of noise, the model was still able to produce complete actions but sometimes failed to sequence those actions correctly, resulting in omission, intrusion and perseveration errors. Action slips tended to occur at branch (or decision) points in the sequence-a time at which additional control is required to prevent the model slipping away from the target action into similar but erroneous sequences afforded by the perceptual input. The model was able to recover from slips, returning to the correct action sequence over ensuing steps following an error.

A stronger level of noise was used to replicate action disorders following neurological damage-a condition generally referred to as action disorganisation syndrome (ADS). Under these conditions, the model became increasingly reliant on actions that were common to a range of different action sequences. Extended periods of "toying" behaviour were produced, for example, in which objects were repetitively picked up and put back down without being used. Actions that had a more specific role in particular sequences - such as pouring, which was only applicable when adding milk - were produced less frequently, resulting in a high omission rate. Rather than occurring at branch points between completed sequences, errors began to infiltrate subtask boundaries, which caused action to become increasingly fragmented. The highly disruptive effect of increased noise meant that the model's attractor dynamics were less frequently able to recover a task sequence once it had been abandoned in favour of an erroneous action.

In the present study we examined the consequences of SA for naturalistic object use. Previous studies with this patient group have demonstrated that a failure of semantic control causes patients to fail not only word and picture-based semantic tasks but also those assessing non-verbal semantic knowledge through single object use. We anticipated that patients with SA would perform even more poorly on naturalistic action tasks, which place a particularly high demand on the controlled use of non-verbal semantic knowledge. Further, errors made by patients during everyday action sequences were likely to reflect the deregulated nature of their semantic cognition. If correct, it should be possible to find common error patterns between SA patients' action sequences and those produced by Botvinick and Plaut's (2004) PDP model of action. 
Table 1

Background details for comprehension impaired stroke aphasic patients.

\begin{tabular}{|c|c|c|c|c|c|c|c|c|}
\hline Case & Age & Sex & $\begin{array}{l}\text { Education } \\
\text { (leaving age) }\end{array}$ & $\begin{array}{l}\text { Neuroimaging } \\
\text { summary }\end{array}$ & L frontal lesion & $\begin{array}{l}\text { L temporo-parietal } \\
\text { lesion }\end{array}$ & Aetiology of CVA & Aphasia type \\
\hline $\mathrm{HN}$ & 78 & M & 14 & L occipital-temporal & $x$ & $\sqrt{ }$ & & Anomic/TSA \\
\hline SC & 76 & M & 16 & $\begin{array}{l}\text { L occipital-temporal } \\
\text { (and R frontal-parietal) }\end{array}$ & $x$ & $\sqrt{ }$ & Haemorrhage & Anomic/TSA \\
\hline NY & 63 & M & 15 & $\begin{array}{l}\mathrm{L} \\
\text { frontal-temporal-parietal }\end{array}$ & $\sqrt{ }$ & $\sqrt{ }$ & & Conduction \\
\hline PG & 59 & M & 18 & $\begin{array}{l}\text { L frontal \& capsular } \\
\text { (CT) }\end{array}$ & $\sqrt{ }$ & a & Subarachnoid haemorrhage & TSA \\
\hline JD & 81 & M & 16 & $\begin{array}{l}\text { Compression of } \mathrm{L} \\
\text { lateral ventricle, } \\
\text { infarction of putamen } \\
\text { and internal capsule }\end{array}$ & a & a & Slightly haemorrhagic & Mixed transcortical \\
\hline ME & 36 & $\mathrm{~F}$ & 16 & L occipital-temporal & $x$ & $\sqrt{ }$ & Subarachnoid haemorrhage & TSA \\
\hline BB & 55 & $\mathrm{~F}$ & 16 & $\begin{array}{l}\text { L frontal and capsular } \\
\text { (CT) }\end{array}$ & $\sqrt{ }$ & $\sqrt{ }$ & Subarachnoid haemorrhage & Mixed transcortical \\
\hline LS & 71 & M & 15 & $\begin{array}{l}\mathrm{L} \\
\text { temporal-parietal-frontal }\end{array}$ & $\sqrt{ }$ & $\sqrt{ }$ & & TSA \\
\hline
\end{tabular}

a Not mentioned on scan report.

\section{Method}

\subsection{Participants}

Eight patients with aphasia following a stroke were recruited from speech and language therapy services and stroke clubs in the Manchester area. Patients were invited to participate in the study if they failed both word and picture versions of the semantic association Camel and Cactus Task (Bozeat et al.,2000). All the patients had experienced a CVA at least a year before the study, which caused chronic impairment. Of the patients we were able to scan (6/8 cases), the left inferior prefrontal cortex and/or left temporoparietal cortex were the most common areas of damage (see Table 1). Where scans were not available (JD and PG), lesions were identified from neuroradiological reports meaning the focal site of cortical damage was less clear in these cases.

The performance of the SA group was compared with that of nine healthy, age and education matched control participants (mean age at recruitment: controls $=65.6$ years, SA patients $=64.9$ years, $t(15)<1$; mean years in education : controls $=10.56$ years, SA patients $=10.75, t(15)<1$ ). The control participants were suitable to take part in the study if they obtained scores within the normal range on the Mini Mental State examination (all scores $>28$ out of a possible 30, Folstein, Folstein, \& McHugh, 1976).

\subsection{Background neuropsychology}

\subsubsection{General neuropsychology}

The SA patients were tested on a range of general neuropsychological tasks, including: forward and backwards digit span, (Wechsler, 1987), non-word and realword repetition from the PALPA (tests 8 and 9, Kay, Lesser, \& Coltheart, 1992) and the Visual Object Space Perception battery (Warrington \& James, 1991). The following tasks were used to assess attentional and executive processing: the Wisconsin card sorting task (WCST, Milner, 1964; Stuss et al., 2000), the Elevator counting subtests of the Test of Everyday Attention (with and without distraction, Robertson, Ward, Ridgeway, \& Nimmo-Smith, 1994), the Brixton Spatial Rule Attainment Task (Burgess \& Shallice, 1996) and the Coloured Progressive Matrices test of non-verbal reasoning (Raven, 1962).

\subsubsection{Semantic memory assessmen}

The SA patients were assessed on a range of tests of semantic knowledge. The Pyramids and Palm Trees test examined semantic associative knowledge (PPT Howard \& Patterson, 1992). This task required participants to decide which of two response options (e.g., palm tree/pine tree) was most closely related to the probe item (e.g., pyramid). Each SA patient completed the task in both picture and word formats. Category fluency was assessed for six categories (animals, birds, fruit, household items, tools and vehicles) and compared to letter fluency (F, A, and S).

Patients were also tested on a battery of three tasks that assessed knowledge of 64 items drawn from the same six semantic categories. The tasks were:

(i) The Camel and Cactus test (CCT, Bozeat et al., 2000). This is an association task similar to the PPT (described above). Participants were required to select which of four response options was related to a probe item. The test was administered in both word and picture formats.

(ii) Spoken word-to-picture matching. For this task, participants selected which picture, from an array of ten possible response options, corresponded to a spoken label given by the experimenter. All of the possible responses on a particular trial were drawn from the same semantic category. (iii) Picture naming. Participants were presented with a line drawing of each item in the battery $(n=64)$ and asked to give its name verbally.

\subsubsection{General praxis}

Participants' ability to imitate ten meaningless gestures was used as a measure of general praxis (Goldenberg, 1996). Two points were awarded if the gesture was correct on the first attempt and no points were awarded if the gesture was not produced correctly following two attempts.

\subsection{Naturalistic action task (Schwartz et al., 2002)}

All participants were tested on the Naturalistic Action Task (NAT, Schwartz et al., 2002), which assessed their ability to perform everyday action sequences. All demonstrations were video recorded for subsequent analysis

\subsubsection{Design}

Three subtasks that varied in their degree of complexity depending on how many goals had to be completed and whether or not distracter items were present in the experimental setup. Subtask 1 asked participants to make a cup of instant coffee with milk and sugar and a slice of toast with butter and jam. Only the items needed to complete the task were available for participants to use. Subtask 2 had the sole aim of wrapping a gift as a present. In addition to the items required to complete the task, however, a number of semantically related distracter items were embedded within the array of items available to use. For example, secateurs were provided in addition to scissors. Subtask 3 was the most complex task as participants not only had to coordinate a dual-task scenario (packing a child's lunchbox and a bag for school) but also omit a large number of semantically related distracters from their action sequences.

\subsubsection{Procedure}

Participants were given standard task instructions (delivered verbally) and a picture of the task's completed goal state (e.g., a picture of a wrapped gift). All standardised procedures for the NAT were followed; objects were arranged in the same position for each participant and a standard cueing procedure was used when necessary. An unlimited amount of time was allowed to complete the task but the experimenter terminated the test if the participants exceeded a time limit of at least 3 min of non-progressive behaviour.

Some of the SA patients had a degree of hemiplegia affecting their right arm. In order to control for any possible implications of hemiplegia, four of the nine control participants were asked to use only their left arm when completing the task. Points were not deducted from either the SA patients or the control participants under sham-hemiplegia conditions if an error was made that was clearly related to thei physical limitations. For example, one patient with right-sided hemiplegia ripped the wrapping paper following an unsuccessful attempt to use the scissors with their left hand; this was not counted as an error.

\subsubsection{Scoring}

All demonstrations were given a basic accomplishment score, which reflected the number of compulsory steps that were completed by the participant-regardless of whether those steps were achieved immediately or following several incorrect actions (maximum accomplishment score for each subtask $=6$, total possible accomplishment score $=18$ ). Each demonstration was also awarded an error score which could comprise errors from the following categories (originally described by Schwartz et al., 2002): (1) step omissions, in which an isolated step was left out of a demonstration; (2) anticipation/omissions, in which an upcoming step was 
performed in advance of an intervening action-irrespective of whether or not the omitted action was ever performed; (3) perseverations of previous actions; (4) reversal of steps or subtasks such that they were performed in the incorrect order; (5) substitutions of a target object with a semantically related distracter; (6) gesture errors, which took the form of an incorrect action used with a correct object; (7) spatial estimations, in which a participant made a gross under/over estimation of the amount of an item required (e.g., far too much wrapping paper); (8) tool/implement omissions; (9) action additions, in which the participant performed an action that was not readily interpretable as a step in the task; and (10) quality errors, which referred to incidents of inexact performance (e.g., packing all the biscuits into the lunchbox). Errors were coded by two raters who demonstrated good inter-rater reliability (kappa $=.88, p<.001)$

The formal scoring system, described above, was a useful tool for the initial assessment of action disorganisation in our patient group. As this system was designed to capture a range of action disorders including ideomotor apraxia, however, it was necessary to make four amendments that accounted for specific error types made by SA patients. Firstly, action addition errors were divided into true additions (defined as above) and toying errors, which were aimless manipulations of objects including picking an object up and putting it back down without making use of that item. Secondly, the formal scoring system only registered a perseveration error if the same object was erroneously used in a repetitive manner (e.g. adding more than one spoonful of coffee). Although the SA patients rarely made perseverations of this type, they did incorrectly reproduce the same action with different objects (e.g., repeatedly packing incorrect objects into the school bag). It was necessary, therefore, to adjust the definition of perseverations to include repetition of the same action on different recipients. In addition, we developed a measure of behavioural fragmentation by examining the proportion of all errors (except omissions) that occurred within an action stream versus those that were 'independent'. Whereas 'within-stream' errors arose within a successfully completed subtask, an 'independent error' reflected a serious interruption in the action stream such that the task was deemed to have been abandoned in favour of non-progressive behaviour. Finally, we created a hesitancy score to account for the number of pauses made by participants during their demonstrations. Participants were considered to have made a hesitancy error if they paused for more than $3 \mathrm{~s}$ without attempting to make the next action.

\section{Results}

\subsection{Background neuropsychology}

\subsubsection{General neuropsychology}

The SA patients spanned a range of impairment severities on general neuropsychological tasks (see Table 2). Two patients (LS and ME) performed particularly poorly on the VOSP test of visuospatial processing, failing three and four subtests respectively. LS and ME also performed poorly on the Raven's Coloured Progressive Matrices test of non-verbal reasoning (10th and 5th percentile scores respectively). All other patients obtained scores at the 50th percentile, except JD who scored at the 95th percentile. A range of tasks was used to assess attentional/executive skills, including: the WCST (Milner, 1964; Stuss et al., 2000), Brixton spatial anticipation (Burgess \& Shallice, 1996) and the elevator counting tests from the TEA (Robertson et al., 1994). All participants obtained impaired scores in at least one of these tasks (except JD, who scored just within the normal range on two tasks; see Table 2). The patients were varied in their performance on forwards and backwards digit span tasks. LS failed both tasks and 3 patients were impaired on at least one digit span task (BB, NY, and HN).

\subsubsection{Semantic memory}

The SA group were impaired on all tasks in the semantic battery, including: picture naming, word-picture matching, associative matching tasks (PPT/CCT), generative naming tasks (category and letter fluency), and matching environmental sounds with words and pictures (see Table 3). None of the patients passed more than one of these tasks, except SC who scored within the normal range on the PPT (words and pictures) and letter fluency task, but obtained impaired scores on more demanding versions of these tasks (CCT words and pictures and category fluency respectively).

\subsubsection{General praxis}

Imitation of meaningless gestures was performed well with $85 \%$ accuracy or greater obtained by all participants except LS, whose vague movements achieved an impaired score of $10 \%$. Although JD was not examined on the meaningless gesture imitation task, accurate production of gestures to verbal command (e.g., 'salute', pp. 140-141, Strub \& Black, 1987) suggests that the patient's general praxis was good.

\section{Naturalistic action task}

As described in Section 2, this task was assessed on two scales: accuracy and error. The accuracy score reflected the number of steps in the task that were successfully completed by the participant, irrespective of how many errors were made in accomplishing these steps. The error score included all errors made by participants during the task even if these errors were corrected before completion of the task; individual error types are considered in separate analyses below.

\subsubsection{Accuracy}

The SA patient group performed more poorly on the NAT than the control participants (see Fig. 1 and Table 4). A repeated measures analysis of variance (ANOVA) showed significant main effects of task (i.e., subtask $1 / 2 / 3 ; F(2,30)=8.50, p=.001$ ) and group (SA patients/control participants; $F(1,15)=31.10, p<.001$ ) but no significant interaction between the two $(F(2,30)=1.16$, n.s.). The patients

Table 2

Background neuropsychological assessments.

\begin{tabular}{|c|c|c|c|c|c|c|c|c|c|c|c|}
\hline Task & Max & Normal cut-off & SA average & $\mathrm{HN}$ & $\mathrm{SC}$ & NY & PG & JD & ME & $\mathrm{BB}$ & LS \\
\hline VOSP dot counting & 10 & 8 & 8.2 & 8 & 10 & 10 & $5^{\mathrm{a}}$ & 10 & $3^{\mathrm{a}}$ & 10 & $6^{\mathrm{a}}$ \\
\hline VOSP position discrimination & 20 & 18 & 17.6 & 19 & $17^{\mathrm{a}}$ & 20 & 20 & 20 & $15^{\mathrm{a}}$ & 18 & $16^{\mathrm{a}}$ \\
\hline VOSP number location & 10 & 7 & 7.4 & 9 & 10 & 10 & 9 & 10 & $2^{\mathrm{a}}$ & 8 & 8 \\
\hline VOSP cube analysis & 10 & 6 & 5.3 & $4^{\mathrm{a}}$ & 9 & $5^{a}$ & 10 & 10 & $4^{\mathrm{a}}$ & $2^{a}$ & $4^{\mathrm{a}}$ \\
\hline Raven's coloured matrices (percentiles) & & & & 50 & 50 & 50 & 50 & $>95$ & $<5^{\mathrm{a}}$ & 50 & 10 \\
\hline WCST (number of categories) & 6 & $1^{\mathrm{b}}$ & 1.2 & 6 & 6 & 2 & $0^{\mathrm{a}}$ & 1 & $0^{\mathrm{a}}$ & 1 & $0^{\mathrm{a}}$ \\
\hline Brixton spatial anticipation (correct) & 54 & 28 & 18 & $26^{\mathrm{a}}$ & $25^{\mathrm{a}}$ & 34 & $26^{a}$ & 28 & $11^{\mathrm{a}}$ & $23^{a}$ & $14^{\mathrm{a}}$ \\
\hline TEA: counting without distraction & 7 & 6 & 4.5 & 7 & 7 & $3^{\mathrm{a}}$ & $3^{a}$ & 7 & 7 & $4^{\mathrm{a}}$ & $3^{\mathrm{a}}$ \\
\hline TEA: counting with distraction & 10 & 3 & 2.13 & 9 & $1^{\mathrm{a}}$ & $2^{\mathrm{a}}$ & $0^{\mathrm{a}}$ & 6 & 9 & $0^{\mathrm{a}}$ & $2^{\mathrm{a}}$ \\
\hline Digit span: forwards & - & 5 & 3.7 & $4^{\mathrm{a}}$ & 6 & $3^{\mathrm{a}}$ & 6 & 5 & 6 & 5 & $4^{\mathrm{a}}$ \\
\hline Digit span: backwards & - & 2 & 1.8 & 3 & 2 & 2 & 2 & 2 & 3 & $0^{\mathrm{a}}$ & $1^{\mathrm{a}}$ \\
\hline \multicolumn{12}{|l|}{ PALPA 8 non-word repetition total } \\
\hline & 30 & & & 11 & 26 & 12 & 22 & 22 & 28 & 25 & 27 \\
\hline PALPA 9 real word repetition total & 80 & 73 & & $69^{a}$ & 78 & $65^{\mathrm{a}}$ & 73 & 74 & 80 & 77 & 77 \\
\hline
\end{tabular}

Patients arranged in order of their semantic skill score generated from all task scores in the semantic battery (see Section 2).

a Impaired scores (less than two standard deviations below mean)

b Cut-off for 50-74-year olds (regardless of educational level). 
Table 3

Semantic tests.

\begin{tabular}{|c|c|c|c|c|c|c|c|c|c|c|c|}
\hline Test & Max & Control mean (SD) & Patient mean & HN & SC & NY & PG & JD & ME & BB & LS \\
\hline Picture PPT & 52 & $51.2(1.4)$ & 40.6 & 44 & 50 & 47 & 42 & 46 & 29 & 41 & 31 \\
\hline Word PPT & 52 & $51.1(1.1)$ & 41.5 & 49 & 51 & 42 & 43 & NT & 39 & 35 & 39 \\
\hline Letter fluency & - & $44.2(11.2)$ & 7.1 & 19 & 24 & 5 & 2 & 5 & 14 & 0 & 8 \\
\hline Category fluency & - & $95.7(16.5)$ & 20.5 & 52 & 17 & 25 & 4 & 27 & 25 & 13 & 11 \\
\hline Naming & 64 & $62.3(1.6)$ & 28 & 50 & 28 & 55 & 46 & 49 & 5 & 10 & 5 \\
\hline Word-picture & 64 & $63.7(.5)$ & 53.2 & 50 & 59 & 60 & 58 & 64 & 50 & 54 & 37 \\
\hline Picture CCT & 64 & $58.9(3.1)$ & 36.8 & 54 & 46 & 36 & 44 & 38 & 13 & 38 & 16 \\
\hline Word CCT & 64 & $60.7(2.06)$ & 38.8 & 54 & 56 & 39 & 40 & 38 & 34 & 30 & 16 \\
\hline Sound-picture & 48 & $41.2(2.5)$ & 30 & 36 & 32 & 28 & 33 & 23 & 33 & 26 & 27 \\
\hline Sound-word & 48 & $40.8(3.8)$ & 28 & NT & 32 & 34 & 25 & 26 & 35 & 27 & 17 \\
\hline Spoken word-picture & 48 & $47.8(0.6)$ & 38.7 & 45 & 41 & 44 & 47 & 46 & 40 & 33 & 35 \\
\hline
\end{tabular}

Patients arranged in order of their semantic skill score generated from all task scores in the semantic battery (see Section 2 ). NT = not tested.

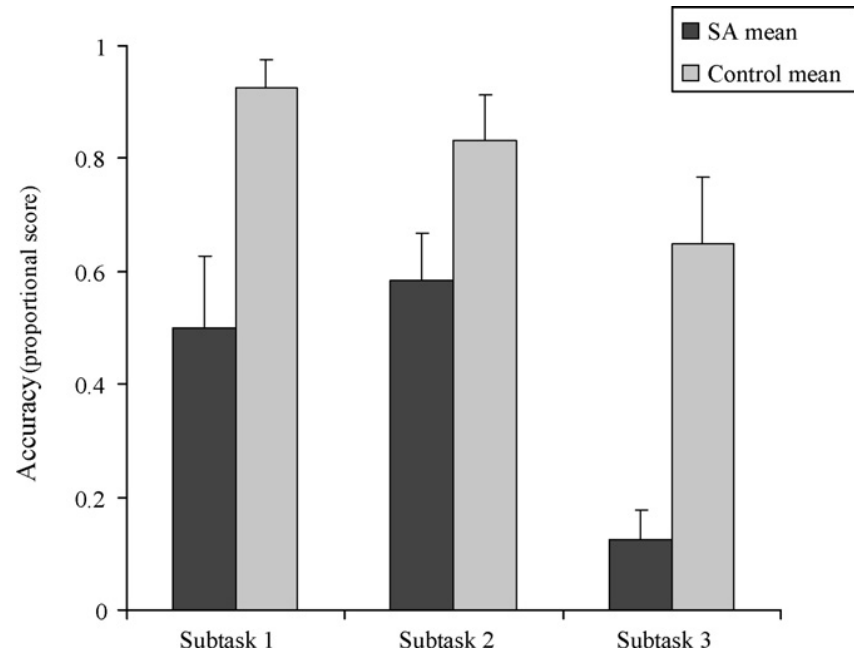

Fig. 1. Accuracy scores for the Naturalistic Action Task (NAT). Error bars $=$ standard error of mean.

performed worse than the control participants on all subtasks $(t(15)$ 2.18-4.01, $p<.045)$. The SA group obtained equivalent accuracy scores for subtasks 1 and $2(t(7)<1)$ but performed significantly worse on subtask 3 (compared to subtask 1: $t(7)=2.61, p=.035$; compared to subtask 2: $t(7)=4.08, p=.005)$. The control group obtained significantly lower accuracy scores on subtask 3 than subtask $1(t(8)=2.5, p=.037)$.

\subsubsection{Total error score}

In line with the basic accuracy scores, the patients made many more errors (when all error types were considered together) than control participants (see Fig. 2). A repeated measures ANOVA showed significant main effects of task $(F(2,30)=13.34, p<.001)$ and group $(F(1,15)=34.64, p<.001)$ and a significant interaction between the two $(F(2,30)=4.3, p=.023)$. The patients made more errors than the control participants on all three subtasks

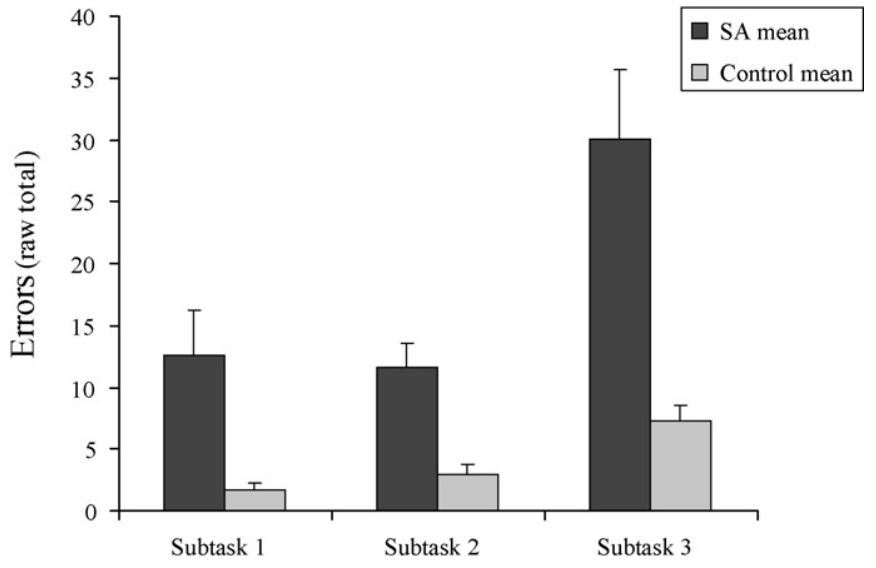

Fig. 2. Total error scores for the NAT. Error bars = standard error of mean.

$(t(15)=3.06-4.2, p<.005)$. Each group made equivalent numbers of errors in subtasks 1 and 2 but made many more errors in subtask 3 (SA patients; $t(7)>2.52, p<.04$, control participants; $t(8)>4$, $p<.004)$. This indicates that individual factors placing demand on control processes (i.e., dual task coordination/presence of distracters tested in subtasks 1 and 2 respectively) did not impact error rate differently from one another but their combined effect (in subtask 3) elicited significantly more errors than either factor in isolation.

\subsubsection{The influence of hemiplegia}

Patients: The SA patients were assessed on four hemiplegia indicators: limb coordination, strength, proprioception and skin sensation (light and sharp touch, see McLeod \& Lance, 1989). Each patient was awarded a score out of five for limb equivalence (where a low score indicated more severe hemiplegia, see Table 4). The degree of patients' hemiplegia did not correlate with overall accuracy or error scores obtained on the NAT $(r<.65, p>.08)$.

Table 4

Accuracy scores for the NAT and hemiplegia limb equivalence scores.

\begin{tabular}{|c|c|c|c|c|c|c|c|c|c|c|c|}
\hline \multirow[t]{2}{*}{ Task } & \multirow[t]{2}{*}{ Max } & \multirow[t]{2}{*}{ Control mean } & \multirow[t]{2}{*}{ SA mean } & \multicolumn{8}{|c|}{ SA patients } \\
\hline & & & & $\mathrm{HN}$ & SC & NY & PG & JD & ME & BB & LS \\
\hline \multicolumn{12}{|l|}{ NAT } \\
\hline Subtask 1 & 6 & 5.5 & 3 & 2 & 4 & 2 & 6 & 6 & 2 & 2 & 0 \\
\hline Subtask 2 & 6 & 5 & 3.5 & 4 & 6 & 2 & 2 & 4 & 2 & 4 & 4 \\
\hline Subtask 3 & 6 & 3.9 & 0.75 & 2 & 0 & 2 & 1 & 0 & 0 & 1 & 0 \\
\hline Hemiplegia limb equivalence score ${ }^{a}$ & 5 & - & - & 5 & 5 & 0 & 2 & 3 & 4 & 4 & 3 \\
\hline
\end{tabular}

a Four hemiplegia indicators were used to generate a score of limb equivalence (limb coordination, strength, proprioception and skin sensation, see McLeod \& Lance, 1989). A low score indicates more severe hemiplegia. Patients arranged in order of their semantic skill score generated from all task scores in the semantic battery (see Section 2 ). 
Control participants: In order to simulate the effects of hemiplegia experienced by some SA patients, four control participants completed the NAT under sham-hemiplegia conditions (i.e., using only their left arm); their performance was compared with that of control participants who used both hands during the task. In a repeated measures ANOVA assessing accuracy scores, there was a significant main effect of subtask (i.e., subtask $1 / 2 / 3 ; F(2,14)=4.2$, $p=.036)$, a main effect of group that approached significance (i.e., both hands/sham-hemiplegia; $F(1,7)=5.15, p=.058$ ) as well as an interaction between the two $(F(2,14)=3.66, p=.053)$. The two groups obtained equivalent accuracy scores for subtasks 1 and 2 $(t(7)<1.9$, n.s.) but participants under sham-hemiplegia conditions obtained lower accuracy scores for subtask $3(t(7)=2.77, p=.027)$. When error scores were considered (including all error types) there was a significant main effect of subtask $(F(2,14)=12.9, p=.001)$ but no effect of group $(F(1,7)=.10$, n.s. $)$ and no group by subtask interaction $(F(2,14)=1.07$, n.s. $)$.

Taken together, these results suggest that hemiplegia is unlikely to make a significant contribution to participants' performance on the NAT. Patients with SA showed no association between their naturalistic object use and degree of hemiplegia. Further, the effect of sham-hemiplegia on control participants' performance was limited; an increased tendency to omit task steps was only observed in subtask 3, which resulted in a lower accomplishment score. However, error scores were not affected. Consequently, the presence of hemiplegia/sham-hemiplegia will not be considered further in subsequent analyses.

\subsection{Naturalistic object use summary}

The SA patients showed marked impairment in their ability to demonstrate naturalistic object use, which went beyond that expected as a result of everyday slips of action. Not only were patients less likely than control participants to carry out essential steps in a task, they were also more likely to incorporate errors in their action sequences. The following analyses will probe the nature of the errors made by the SA patients and controls. Error analysis will take place in two stages; firstly the formal error coding system described by Schwartz et al. (2002) will be used to explore whether the errors made by the two groups were distributed in the same proportions across different error categories (including the additional 'hesitancy' category). While this scoring system provided a useful preliminary tool with which to assess our patient group, its application with a range of action disorders, including motor disorders such as ideomotor apraxia, means that it was unable to capture all important aspects of the behaviour exhibited by SA patients. The second stage of analyses will, therefore, further explore qualitative characteristics of action disorders specifically observed in SA.

\subsection{Qualitative characteristics of performance on the NAT}

\subsubsection{Error analysis (Schwartz et al., 2002)}

When proportional error scores were considered, the distribution of errors across different categories (as described in Section 2, see also Schwartz et al., 2002) was largely similar for the patient and control groups. Both groups committed mostly step omission and action addition errors which together accounted for over $50 \%$ of errors in both groups (see Fig. 3). Hesitancy, quality and anticipation/omission errors were also common for both groups whereas perseverations, reversals, substitutions, gestures spatial estimations and tool/implement omissions were rarely made by either group. When the results from all three subtasks were taken together, proportional error scores were equivalent for all error types in the two groups $(t(7-15)<1.76$, n.s.) except for tool omissions, which made up a larger proportion of the SA group's errors than the control group $(t(7)=2.42, p=.046)$. Likewise, the propor-

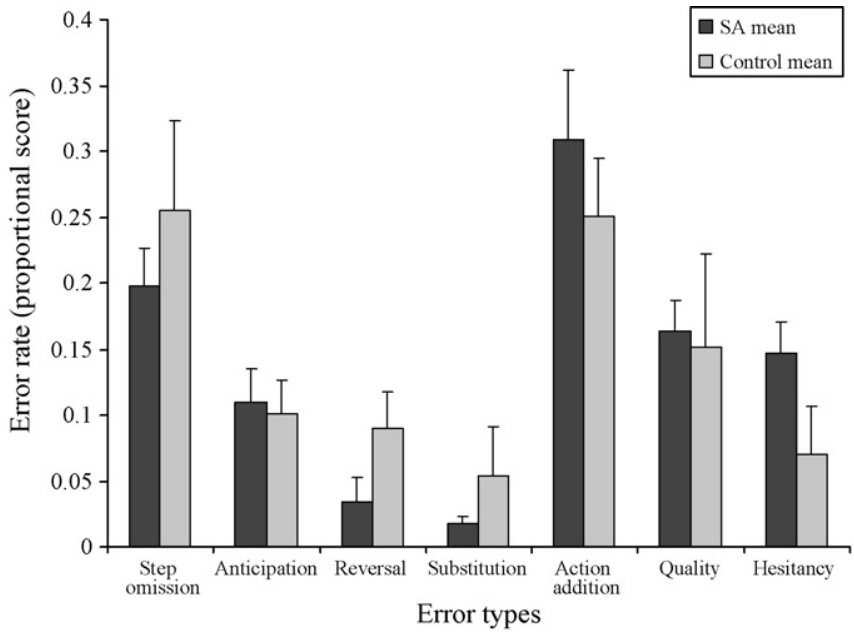

Fig. 3. Proportional breakdown of errors. Some error categories removed where very few errors were made (perseveration, gesture, spatial estimation and tool/implement omission errors). Error bars $=$ standard error of mean.

tion of each error type did not differ significantly for the two groups for any of the individual subtasks $(t(15)<2.1, p>.053)$.

\subsubsection{Additional error types}

In addition to the formal error coding system, which is suitable for use with a range of patients with action disorders, we noted some different error types, which were characteristic for the SA patients; these are discussed below.

\subsubsection{Action additions}

A true action addition took the form of an action that was unrelated to the overall aim of the task but had a clear local purpose. For example, PG made a number of true additions by unpacking items from the lunchbox, which constituted an action that had a clear immediate aim but that was not legitimately required to move the task towards its eventual aim. Botvinick and Plaut's (2004) computational model also made addition errors during naturalistic action, but many of them differed qualitatively from true action additions. Under strong levels of noise the model made many "toying" errors in which additional actions were made that served no clear purpose. For example, an object might be picked up, held and possibly manipulated, and put back down without being used. We therefore explored the nature of the action additions made by our participants.

Both the SA patients and control participants made many action addition errors (see Fig. 3). For the SA patients, those action additions were dominated by toying errors ( $67 \%$ of all action additions), which were significantly greater in number than true action additions (see Fig. 4: $t(7)=2.6, p=.035$ ). The control participants also made more toying errors than true action additions ( $58 \%$ of action errors were toying errors), but the difference was not significant $(t(8)=.79$, n.s.). In a repeated measures ANOVA there was no significant interaction between error type (true addition/toying) and group (control participants/SA patients; $F(1,15)=.48$, n.s.).

\subsubsection{Perseverations}

According to the formal scoring system, a perseverative error occurred when an action was inappropriately repeated in a recipient-focused fashion; such as repeated packing of biscuits into the lunchbox. Although participants in this study did not perseverate actions towards the same recipient, they did inappropriately reproduce the same action on different objects. For example, JD perseverated on a single action by continuously packing distracter 


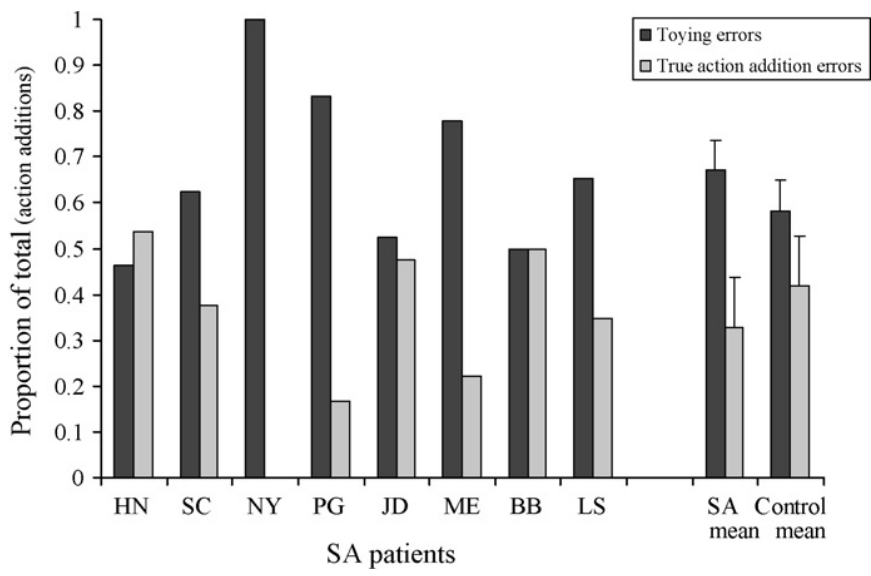

Fig. 4. Proportion of action additions that were toying errors. Patients arranged in order of their semantic skill score generated from all task scores in the semantic battery (see Section 2). Error bars = standard error of mean.

items into the school bag, including items such as a pair of tongs, a toothbrush and an envelope. For the following analysis, we therefore identified incidents of action - but not recipient - specific perseverations.

Perseverations were present in at least one subtask for all SA patients except $\mathrm{BB}$, whose tendency to make step omission errors meant that she had less opportunity to make a perseverative error (31\% of all errors made by BB were step omissions compared to an average of $18 \%$ for all other SA patients). By contrast, none of the control participants were found to inappropriately repeat the same action.

\subsubsection{Fragmentation of behaviour}

In addition to the different types of errors made during naturalistic object use, the extent to which those errors caused behaviour to become disorganised has been identified as an important measure of naturalistic action disorders (Schwartz, Reed, Montgomery, Palmer, \& Mayer, 1991). In a detailed examination of action disorganisation syndrome following brain damage, Schwartz et al. (1991) reported that highly disorganised action was characterised by a greater number of actions occurring outside the boundaries of a completed subtask. These errors were described as 'independent' actions (Schwartz et al., 1991).

In the present analysis we used Schwartz et al.'s (1991) principles as a foundation for developing a measure of behavioural fragmentation. We categorised errors made by participants as either 'independent' or 'within-stream'. Within-stream errors were those that occurred within the context of a successfully completed subtask. This type of error did not cause significant deviation from the current subtask. For example, if a participant successfully added coffee to the hot water during the coffee making task, but used the fork instead of the spoon, the tool substitution would be classed as a within-stream error. An independent error, conversely, occurred outside the boundaries of a completed subtask (e.g., abandoning an incomplete subtask in favour of aimless toying with the objects). The proportion of errors that were independent gave a measure of the readiness with which subtasks were abandoned in favour of non-progressive action. We assessed the proportion of errors that were independent versus those that occurred within-stream. All types of error were included in the analysis excluding errors of omission, which cannot be localised to a particular point in the action stream.

When the number of independent versus within-stream errors was compared across the two groups (SA patients/control participants) in a repeated measures ANOVA there was no main effect

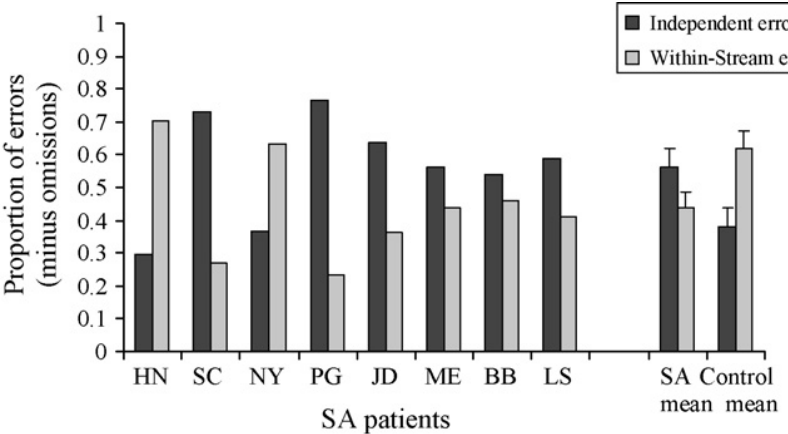

Fig. 5. All errors (minus omissions) broken down into stream and independent errors. Patients arranged in order of their semantic skill score generated from all task scores in the semantic battery (see Section 2). Error bars = standard error of mean.

of error type $(F(1,15)=2.39$, n.s., see Fig. 5$)$ but there was a significant main effect of group $(F(1,15)=29.81, p .001)$ and a group by error type interaction $(F(1,15)=4.6, p=.049)$. The SA patients made equivalent numbers of independent and within stream errors $(t(7)=1.8$, n.s.), while the control participants made proportionally fewer independent errors than within-stream errors $(t(8)=2.18$, $p=.06$ ), which suggests that everyday action had become substantially more disorganised in SA than would be expected as a consequence of everyday action slips.

\subsubsection{Task correlations}

Correlations between conceptual tasks and naturalistic action: Principal components analysis was used to extract a single semantic skill score for each patient based on their performance across all tasks used to assess their semantic memory (see Section 2). The patients' semantic skill scores were found to correlate with their overall accuracy scores on the NAT $(r=.70, p=.03$, all correlations are one-tailed). Of all the tasks in the semantic battery, the following were highly correlated with accuracy on the NAT: word-picture matching, the Camel and Cactus test (both word and picture versions) and the picture version of the Pyramids and Palm Trees task $(r=.69-.83, p<.03)$. Near-significant correlations also occurred for naming and the word version of the Pyramids and Palm trees task $(r=.52-.61, p<.094)$.

\subsubsection{Correlations between executive tasks and naturalistic action}

Overall accuracy correlated with an executive skill factor derived, using the same method as described above, from the WCST and Raven's Coloured Progressive Matrices task $(r=.7, p=.027)$.

\subsubsection{Correlations between conceptual tasks and specific errors made during naturalistic action}

Patients semantic skill scores correlated negatively with step omission errors in subtasks 1,2 and overall $(r=-.80, p=.012)$, as well as action additions (all additions considered together, $r=-.64$, $p=.03$ ) and anticipation errors in subtask $1(r=-.69, p=.03)$.

\subsubsection{Correlations between executive skill and specific errors made during naturalistic action}

Significant negative correlations occurred between the executive skill factor and step omission errors in subtasks 1 and $2(r=-.63$ to $-.69, p<.046$ ) and action addition errors in subtask 1 (all action additions considered together, $r=-.77, p=.013$ ).

\subsubsection{Correlations between visuo-spatial tasks and naturalistic action}

Accuracy and error scores on the NAT correlated with the cube analysis subtest of the VOSP $(r=.66-.74, p<.04)$, which is per- 
haps unsurprising given the high load placed on executive control processes by this task. Accuracy also correlated with position discrimination and number location $(r=.62-.67, p<.05)$ but not dot counting $(r=.54$, n.s.). SA patients' errors were dominated by action additions and step omissions (together accounting for over $50 \%$ of all errors), but neither error type was associated with performance on the VOSP, except one significant correlation between action additions and cube analysis $(r=.69, p=.03)$. Hence, although visual processing and object use are clearly linked, it is unlikely that the visuo-spatial impairment exhibited by some patients in this study was the primary cause of the errors we observed in naturalistic object use.

\section{Discussion}

This study assessed the impact of impaired semantic control arising in semantic aphasia (SA) on a non-verbal expressive task-naturalistic object use. Patients' performance on everyday action tasks, such as making a cup of coffee, was examined for characteristics of deregulated semantic cognition-previously only observed in picture and word-based tasks and more straightforward single object-use tests (Corbett et al., in press; Jefferies \& Lambon Ralph, 2006; Noonan et al., in press). Patients with SA scored more poorly on the Naturalistic Action Test (Schwartz et al., 2002) than age and education matched control participants; their action sequences were incomplete and disrupted by large numbers of action addition and step omission errors. Control participants also made a number of action slips during their demonstrations, but they were much lower in number than the errors made by the SA group. This study therefore provides converging evidence for a truly multimodal deficit of conceptual knowledge in SA, which not only affects semantic processing in the verbal domain but also infiltrates naturalistic object use. This is a striking finding given that SA patients are most commonly studied in the context of their presenting symptom, profound language impairment. Probing the nature of action disorganisation further revealed a number of characteristics of SA patients' performance that were indicative of a failure of semantic control processes.

SA patients' low accuracy scores in naturalistic object-use and high rates of omission and action addition errors (as well as a smaller number of anticipation errors), correlated with their level of impairment on standard word and picture-based semantic tasks. This finding is concordant with the hypothesis that the control aspect of semantic cognition has been damaged in SA, leading to impaired semantic processing irrespective of input/output modality. Previous studies in the verbal domain have identified qualitative aspects of SA patients' performance on semantic tasks that are indicative of their deregulated semantic processing. In picture naming, for example, performance is improved by decreasing the burden on executive control processes with highly constraining phonemic cues and also impaired by distracting miscues. These effects are not observed in conditions in which multimodal semantic impairments arise through degradation of semantic representations, such as semantic dementia (Jefferies \& Lambon Ralph, 2006; Jefferies et al., 2008). The NAT manipulated demand on controlled use of non-verbal conceptual knowledge with dual-task situations and the presence of semantically related distracting objects. As predicted, SA patients performed significantly more poorly when they had to manage a dual-task scenario and refrain from using distracting objects in the same task. These findings converge with those from the verbal domain by demonstrating that SA patients' naturalistic object-use was sensitive to the load placed on control processes.

The nature of action disorganisation exhibited by the SA group replicated the performance of computational models in which action disorganisation was simulated through damage to control processes. Botvinick and Plaut (2004) developed a parallel distributed processing model of everyday action (described in Section 1 ), in which an action was selected from a range of possible responses activated by a visual or tactile perceptual input. In this model, the action selection process was constrained by the temporal context of that action, which was represented in the recurrent connections of the system's hidden layer. Application of random noise to this control mechanism caused the model's output to become disrupted. At low levels of noise, disruption was minimal and the errors made were characteristic of everyday action slips. Both the model and control participants in the present study made mostly omission errors but were able to overcome these mistakes over ensuing steps, in order to return to a correct action sequence. As noise was gradually increased, the model's behaviour became more disorganised. Although the same types of errors were being made (i.e., mostly step omissions and action additions), they were now infiltrating subtask boundaries rather than largely occurring at the transition of completed subtasks-a time when extra demand is placed on control processes. This characteristic was also observed in our group of SA patients who, unlike control participants, made as many errors outside the context of a completed subtask - indicating action disruption - as they did within successful action sequences. When noise levels became particularly high, the model's attractor dynamics were unable to draw the system back into an action sequence following an error. Consequently, the model produced extended periods of aimless toying behaviour. Similarly, SA patients with lower semantic and executive skill scores were found to make a greater number of omission and action addition errors than patients who scored more highly on these assessments. Many of these action addition errors reflected precisely this type of aimless toying. Although control participants also committed toying errors, which could reflect investigations of objects not previously encountered, they occurred much less frequently than observed in SA. Further, both the SA patients and the model under strong levels of noise demonstrated a tendency to erroneously reproduce the same actions.

The recurrent connectionist approach put forward by Botvinick and Plaut (2004) is not the only model to have replicated routine action. Cooper and Shallice (2000) developed a model of everyday action based on the principles of contention scheduling (first described by Norman \& Shallice, 1986). This model differs from PDP accounts as the hierarchical nature of everyday action sequences is pre-specified in a structured network of action schemas. According to this approach, action goals are fulfilled when a relevant source schema, residing in the schema network, is activated above threshold. There are several different sources of excitation/inhibition that influence schema activation. A source schema will become more highly activated, for example, through bottom-up inputs from a pertinent object present in the visual scene. Once a source schema has been selected, activation spreads across to complementary resource and object networks but also in a top-down fashion to lower level schemas within the same network. These low-level schemas act to fulfil the goals of the schemas at higher levels of the hierarchy. A highly activated schema node has a lateral inhibitory effect on other schemas that share the same goal or are in competition for the same resources. The model's execution is synchronous and cyclical in nature; on each cycle, all activation values in the schema, object and resource networks are updated and selections/deselections made accordingly.

Cooper et al. (2005) applied damage to three components of their hierarchical model to simulate different types of action disorder. Firstly, the relative influence of top-down control over schema activation by source schemas was weakened in favour of an increased contribution from bottom-up environmental sources. Under these conditions the model's behaviour closely resembled 
that of utilisation syndrome, featuring extended periods spent aimlessly toying with the objects. In two further simulations, Cooper et al. applied damage to the schema network and object representation networks to reproduce two disorders characterised by impaired naturalistic object use - action disorganisation syndrome and ideational apraxia respectively. Due to reciprocal connections between the schema and object networks in this model, the consequences of these two simulations were largely indistinguishable. The model made mostly step omission errors, which were particularly prevalent when overall accomplishment levels were low and in the presence of distracting objects. Therefore the contentionscheduling approach also reproduced many characteristics of action disorganisation that we have observed in the context of SA. However, the principles by which damage was applied to Botvinick and Plaut's (2004) PDP model are more compatible with our account of this disorder. In the PDP model, a single source of damage was applied to the hidden layer, which impaired the system's ability to select an appropriate action in a given context. This approach makes close contact with our conception of SA in which damage to the control component of cognition impairs patients' ability to constrain/shape semantic processing in a task/context appropriate fashion. Further, the PDP model uniquely reproduced the recurrent perseverative aspect of SA patients' behaviour.

In this study, the NAT was a useful tool in uncovering impaired non-verbal semantic processing in SA but also revealed qualitative characteristics of patients' deregulated semantic cognition that previous studies have not been able to tap through single object use tasks (e.g., Corbett et al., in press). Similarities between SA patients' performance and that of Botvinick and Plaut's (2004) model of everyday action provide further support for a failure of semantic control underlying this condition. Critically, this study highlights the importance of examining damage to fundamental components of cognition, such as semantic control, that underpin a broad range of verbal and non-verbal activities. Our findings could also have implications for more traditional approaches to apraxia. A range of action disorders that arise in the absence of motor deficits have been identified in apraxia literature, such as action disorganisation syndrome (ADS) and ideational apraxia (IA). Despite the similar neuropsychological profiles in these two conditions they have, for the most part, been addressed by separate studies. This is probably due to their association with different types of neurological damage; whereas ADS is linked with damage in the left inferior frontal gyrus (Humphreys \& Forde, 1998; Schwartz et al., 1995, 1998, 1991), IA is more commonly thought to arise in the context of left temporoparietal damage (De Renzi \& Lucchelli, 1988; Rumiati, Zanini, Vorano, \& Shallice, 2001). In the present study, patients with lesions affecting one or both of these neural sites were indistinguishable in terms of their action disorders. Previous neuropsychological studies in the verbal domain have also noted that patients with lesions in one or both of these sites have similar aphasia profiles and parallel semantic/cognitive impairments (Berthier, 2001; Jefferies \& Lambon Ralph, 2006). Moreover, neuroimaging studies (detailed in Section 1) have shown greater activation in both of these regions when tasks place demands on cognitive control (Demb et al., 1995; Gold \& Buckner, 2002; Noppeney et al., 2004; Thompson-Schill et al., 1997; Wagner et al., 2001). It is possible, therefore, that ADS and IA actually reflect disorders of the same cognitive control system but follow damage to different parts of the distributed neural network underlying action control. Because semantic memory has not been assessed more broadly in previous reports of IA and ADS, however, it is difficult to be sure about how these disorders relate to SA. To explore this idea, future studies could compare the breakdown of naturalistic action in larger groups of patients with lesions in these two regions. Moreover, it would also be interesting to examine performance on the NAT in the context of qualitatively different disorders of semantic cognition-including semantic dementia patients who have degraded semantic representations (see Buxbaum, Schwartz, \& Carew, 1997).

In conclusion, our findings provide converging evidence for the hypothesis that semantic cognition has become deregulated in SA, leading to a multimodal semantic deficit - impairing semantic processing not only in the verbal domain, but also in non-verbal semantic task - naturalistic object use. Our results confirm that damage to the neural network encompassing the left inferior frontal gyrus and left temporoparietal cortex impairs SA patients' ability to perform everyday action tasks. In particular, their action sequences were fragmented by a tendency to omit essential steps and they engaged in extended periods of aimless toying behaviour. This behavioural profile parallels findings from computational models that have sought to reproduce a spectrum of action disorders - ranging from everyday action slips to action disorganisation syndrome - through increasing damage to cognitive control components.

\section{References}

Berthier, M. L. (2001). Unexpected brain-language relationships in aphasia: Evidence from transcortical sensory aphasia associated with frontal lobe lesions. Aphasiology, 15, 99-130.

Botvinick, M., \& Plaut, D. C. (2004). Doing without schema hierarchies: A recurrent connectionist approach to normal and impaired routine sequential action. Psychological Review, 111, 395-429.

Bozeat, S., Lambon Ralph, M. A., Patterson, K., Garrard, P., \& Hodges, J. R. (2000) Non-verbal semantic impairment in semantic dementia. Neuropsychologia, 38 1207-1215.

Bozeat, S., Lambon Ralph, M. A., Patterson, K., \& Hodges, J. R. (2002). When objects lose their meaning: What happens to their use? Cognitive, Affective Er Behavioural Neuroscience, 2, 236-251.

Burgess, P. W., \& Shallice, T. (1996). Bizarre responses, rule detection and frontal lobe lesions. Cortex, 32, 241-259.

Buxbaum, L. J., Schwartz, M. F., \& Carew, T. G. (1997). The role of semantic memory in object use. Cognitive Neuropsychology, 14, 219-254

Chertkow, H., Bub, D., Deaudon, C., \& Whitehead, V. (1997). On the status of object concepts in aphasia. Brain and Language, 58, 203-232.

Coccia, M., Bartolini, M., Luzzi, S., Provinciali, L., \& Lambon Ralph, M. A. (2004) Semantic memory is an amodal, dynamic system: Evidence from the interaction of naming and object use in semantic dementia. Cognitive Neuropsychology, 21, 513-527.

Cooper, R. P., Schwartz, M. F., Yule, P., \& Shallice, T. (2005). The simulation of action disorganisation in complex activities of daily living. Cognitive Neuropsychology, 22, 959-1004

Cooper, R. P., \& Shallice, T. (2000). Contention scheduling and the control of routine activities. Cognitive Neuropsychology, 17, 297-338.

Corbett, F., Jefferies, E., Ehsan, S., \& Lambon Ralph, M. A. (in press). Qualitatively different conceptual impairments in semantic dementia and semantic aphasia: Converging evidence from the non-verbal domain. Brain.

De Renzi, E., \& Lucchelli, F. (1988). Ideational apraxia. Brain, 111, 1173-1185.

Demb, J. B., Desmond, J. E., Wagner, A. D., Vaidya, C. J., Glover, G. H., \& Gabrieli, J. D. E. (1995). Semantic encoding and retrieval in the left inferior prefrontal cortex-A functional MRI study of task-difficulty and process specificity. Journal of Neuroscience, 15, 5870-5878.

Devlin, J. T., Russell, R. P., Davis, M. H., Price, C. J., Wilson, J., Moss, H. E., et al. (2000). Susceptibility-induced loss of signal: Comparing PET and fMRI on a semantic task. Neuroimage, 11, 589-600.

Farah, M. J. (2004). Visual agnosia. Cambridge, MA: US MIT Press.

Folstein, M. F., Folstein, S. E., \& McHugh, P. R. (1976). "Mini-mental state": A practical method for grading the mental state of patients for the clinician. Journal of Psychiatric Research, 12, 189-198.

Franklin, S., Turner, J., Lambon Ralph, M. A., Morris, J., \& Bailey, P.J. (1996). A distinctive case of word meaning deafness? Cognitive Neuropsychology, 13, 1139-1162.

Gloor, P. (1997). The temporal lobe and the limbic system. Oxford: Oxford University Press.

Gold, B. T., \& Buckner, R. L. (2002). Common prefrontal regions coactivate with dissociable posterior regions during controlled semantic and phonological tasks. Neuron, 34, 803-812.

Goldenberg, G. (1996). Defective imitation of gestures in patients with left and right hemisphere damage. Journal of Neurology, 61, 176-180.

Hart, J., \& Gordon, B. (1990). Delineation of single-word semantic comprehension deficits in aphasia with anatomical correlation. Annals of Neurology, 27, 226-231.

Hodges, J. R. Patterson, K. Oxbury, S., \& Funnell, E. (1992). Semantic dementia: Progressive fluent aphasia with temporal-lobe atrophy. Brain, 115, 1783-1806.

Howard, D., \& Patterson, K. (1992). Pyramid and palm trees: A test of semantic access from pictures and words. Bury St Edmunds: Thames Valley Test Company.

Humphreys, G. W., \& Forde, E. M. E. (1998). Disordered action schema and action disorganisation syndrome. Cognitive Neuropsychology, 15, 771-811.

Jefferies, E., Baker, S. S., Doran, M., \& Lambon Ralph, M. A. (2007). Refractory effects in stroke aphasia: A consequence of poor semantic control. Neuropsychologia, 45 , 1065-1079. 
Jefferies, E., \& Lambon Ralph, M. A. (2006). Semantic impairment in stroke aphasia versus semantic dementia: A case-series comparison. Brain, 129, 2132-2147.

Jefferies, E., Patterson, K., \& Lambon Ralph, M. A. (2008). Deficits of knowledge versus executive control in semantic cognition: Insights from cued naming. Neuropsychologia, 46, 649-658.

Kapur, N., Barker, S., Burrows, E. H., Ellison, D., Brice, J., Illis, L. S., et al. (1994). Herpes-simplex encephalitis: Long-term magnetic-resonance-imaging and neuropsychological profile. Journal of Neurology, Neurosurgery and Psychiatry, 57, 1334-1342.

Kay, J., Lesser, R., \& Coltheart, M. (1992). Psycholinguistic assessments of language processing in aphasia (PALPA). Hove, UK: Lawrence Erlbaum Associates.

Lambon Ralph, M. A., Lowe, C., \& Rogers, T. (2007). Neural basis of category-specific semantic deficits for living things: Evidence from semantic dementia. HSVE and a neural network model. Brain, 130, 1127-1137.

Lambon Ralph, M. A., Pobric, G., \& Jefferies, E. (2009). Conceptual knowledge is underpinned by the temporal pole bilaterally: Convergent evidence from rTMS. Cerebral Cortex, 19, 832-838.

Luzzi, S., Snowden, J. S., Neary, D., Coccia, M., Provinciali, L., \& Lambon Ralph, M. A. (2007). Distinct patterns of olfactory impairment in Alzheimer's disease, semantic dementia, frontotemporal dementia, and corticobasal degeneration. Neuropsychologia, 45, 1823-2183.

McLeod, J. G., \& Lance, J. W. (1989). Introductory neurology (2nd edition). Blackwell Scientific Publications.

Milner, B. (1964). Effects of different brain lesions on card sorting: The role of the frontal lobes. Archives of Neurology, 9, 100-110.

Mummery, C. J., Patterson, K., Price, C. J., Ashburner, J., Frackowiak, R. S. J., \& Hodges, J. R. (2000). A voxel-based morphometry study of semantic dementia: Relationship between temporal lobe atrophy and semantic memory. Annals of Neurology, 47, 36-45.

Nestor, P. J., Fryer, T. D., \& Hodges, J. R. (2006). Declarative memory impairments in Alzheimer's disease and semantic dementia. Neuroimage, 30, 10101020.

Noonan, K. A., Jefferies, E., Corbett, F., \& Lambon Ralph, M. A. (in press). Elucidating the nature of deregulated semantic cognition in semantic aphasia: Evidence for the roles of prefrontal and temporoparietal cortices. Journal of Cognitive Neuroscience.

Noppeney, U., Phillips, J., \& Price, C. (2004). The neural areas that control the retrieval and selection of semantics. Neuropsychologia, 42, 1269-1280.

Norman, D. A., \& Shallice, T. (1986). Attention to action: Willed and automatic control of behaviour. In R. Davidson, G. Schwartz, \& D. Shapiro (Eds.), Consciousness and self regulation: Advances in research and theory. Vol. 4 (pp. 1-18). New York: Plenum.

Parker, G. J. M., Luzzi, S., Alexander, D. C., Wheeler-Kingshott, C. A. M., Clecarelli, O., \& Lambon Ralph, M. A. (2005). Lateralisation of ventral and dorsal auditorylanguage pathways in the human brain. Neuroimage, 24, 656-666.

Pobric, G., Jefferies, E., \& Lambon Ralph, M. A. (2007). Anterior temporal lobes mediate semantic representation: Mimicking semantic dementia by using rTMS in normal participants. Proceedings of the National Academy of Sciences of the United States of America, 104, 20137-20141.
Raven, J. C. (1962). Coloured progressive matrices sets $A, A B$, B. London: H.K. Lewis. Robertson, I. H., Ward, T., Ridgeway, V., \& Nimmo-Smith, I. (1994). The test of everyday attention. Flempton: Thames Valley Test Company.

Rogers, T. T., Lambon Ralph, M. A., Garrard, P., Bozeat, S., McClelland, J. L., Hodges, J. R., et al. (2004). Structure and deterioration of semantic memory: A neuropsychological and computational investigation. Psychological Review, 111, 205-235.

Rumiati, R. I., Zanini, S., Vorano, L., \& Shallice, T. (2001). A form of ideational apraxia as a selective deficit of contention scheduling. Cognitive Neuropsychology, 18, 617-642.

Saffran, E. M. (2000). The organization of semantic memory: In support of a distributed model. Brain and Language, 71, 204-212.

Saygin, A. P., Dick, F., Wilson, S. M., Dronkers, N. F., \& Bates, E. (2003). Neural resources for processing language and environmental sounds. Brain, 126, 928-945.

Schwartz, M. F., Buxbaum, L. J., Ferraro, M., Veramonti, T., \& Segal, M. (2002). Naturalistic action test. Bury St Edmunds: Thames Valley Test Company.

Schwartz, M. F., Montgomery, M., Fitzpatrick-DeSalme, E., Ochipa, C., Branch Coslett, H., \& Mayer, N. H. (1995). Analysis of a disorder of everyday action. Cognitive Neuropsychology, 12, 863-892.

Schwartz, M. F., Montgomery, M. W., Buxbaum, L. J., Lee, S. S., Carew, T. G., Branch Coslett, H., et al. (1998). Naturalistic action impairment in closed head injury. Neuropsychology, 12, 13-28.

Schwartz, M. F., Reed, E. S., Montgomery, M., Palmer, C., \& Mayer, N. H. (1991). The quantitative description of action disorganization after brain-damage: A casestudy. Cognitive Neuropsychology, 8, 381-414.

Snowden, J. S., Goulding, P. J., \& Neary, D. (1989). Semantic dementia: A form of circumscribed cerebral atrophy. Behavioural Neurology, 2, 167-182.

Strub, R. L., \& Black, F. W. (1987). The mental status examination in neurology. Philadelphia: F.A. Davis Company.

Stuss, D. T., Levine, B., Alexander, M. P., Hong, J., Palumbo, C., Hamer, L., et al. (2000). Wisconsin Card Sorting Test performance in patients with focal frontal and posterior brain damage: Effects of lesion location and test structure on separable cognitive processes. Neuropsychologia, 38, 388-402.

Thompson-Schill, S. L., D’Esposito, M., Aguirre, G. K., \& Farah, M. J. (1997). Role of left inferior prefrontal cortex in retrieval of semantic knowledge: A reevaluation. Proceedings of the National Academy of Sciences of the United States of America, 94, 14792-14797.

Visser, M., Embleton, K. V., Jefferies, E., Parker, G. J. M., \& Lambon Ralph, M. A. (submitted-a). The anterior temporal lobes and semantic memory clarified: Novel evidence from distortion-corrected spin-echo EPI fMRI.

Visser, M., Jefferies, E., \& Lambon Ralph, M. A. (submitted-b). Semantic processing in the anterior temporal lobes: A meta-analysis of the functional neuroimaging literature. Journal of Cognitive Neuroscience.

Wagner, A. D., Pare-Blagoev, E. J., Clark, J., \& Poldrack, R. A. (2001). Recovering meaning: Left prefrontal cortex guides controlled semantic retrieval. Neuron, 31, 329-338.

Warrington, E. K., \& James, M. (1991). The visual object and space perception battery. Bury St. Edmunds, UK: Thames Valley Test Company.

Wechsler, D. (1987). Wechsler Memory Scale-Revised (WMS-R). New York: Psychological Corporation. 